\title{
Both food habit change in the past and obesity status may influence the association between dietary factors and postmenopausal breast cancer
}

\author{
Emily Sonestedt*, Bo Gullberg and Elisabet Wirfält \\ Lund University, Department of Clinical Sciences Malmö, Building 60 floor 13, CRC entrance 72 UMAS, SE-20502 \\ Malmö, Sweden
}

Submitted 28 February 2006: Accepted 23 August 2006: First published online 5 March 2007

\begin{abstract}
Objective: Valid dietary data are essential when trying to identify whether or not one or more dietary exposures are responsible for disease. We examined diet composition in women who reported dietary change in the past compared with non-changers, and how the associations between dietary factors and postmenopausal breast cancer are influenced by dietary change, obesity status and misreporting of energy.

Design: A population-based prospective cohort study. Data were obtained by a diet history method, anthropometrical measurements and an extensive lifestyle questionnaire including items on past food habit change.

Setting: The Malmö Diet and Cancer (MDC) study, conducted in Malmö, Sweden.

Subjects: A subsample of 12781 women from the MDC cohort recruited from 1991 to 1996. A total of 428 postmenopausal women were diagnosed with incident breast cancer, during 9.2 years of follow-up.

Results: Past food habit changers reported healthier food habits and lower energy intake compared with non-changers, a finding that raises issues regarding possible reporting biases. When excluding diet changers, the trend of increased breast cancer risk across omega-6 fatty acid quintiles was stronger, and a tendency of decreased risk emerged for 'fruit, berries and vegetables'. When excluding individuals with nonadequate reports of energy intake, risk estimates were similar to that of the whole sample. In women with body mass index $<27 \mathrm{~kg} \mathrm{~m}^{-2}$, significant trends of increased breast cancer risk were seen for total fat and omega- 6 fatty acids, and of decreased risk for 'fruit, berries and vegetables'.

Conclusions: This study indicates that both obesity and self-reported past food habit change may be important confounders of diet-breast cancer relationships. The study demonstrates that sensitivity analysis, through stratification, may facilitate interpretation of risk relationships and study results.
\end{abstract}

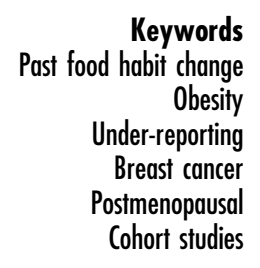

Epidemiology contributes essential knowledge on the relationship between diet and chronic diseases. Nutrition studies commonly use prospective designs so that the reported dietary intakes are not biased by knowledge about the disease ${ }^{1}$. Indeed, there is evidence that biases may exist in dietary self-report that could confound dietdisease relationships even in the context of a prospective study (i.e. the biasing factor may be related to factors that are involved in the process of carcinogenesis) ${ }^{2}$. Moreover, food habits may not be constant over time, and chronic diseases, including cancer, are believed to have long latency periods. Accounting for temporal sequencing of events is complicated by the fact that dietary habits from the distant past may be of greater importance than those close to the presentation of the disease ${ }^{3}$.

Few prospective studies have information on major food habit changes over time. However, such information is essential in order to identify the diet exposure responsible for disease, and to interpret the results of epidemiological studies critically. During the baseline examinations of the Malmo Diet and Cancer (MDC) study, a number of questions on past food habit change were asked, in order to provide an analysis of the most obvious dietary changes. Previous cross-sectional analyses within the MDC study have indicated that individuals with reported past food habit change generally are more obese than individuals not reporting a food habit change ${ }^{4,5}$. Other studies have indicated that obese individuals generally consume less than or the same amount of energy as normal-weight controls ${ }^{6}$, but studies using the doubly labelled water technique have also provided evidence for obesity-specific under-reporting ${ }^{7}$.

The study presented herein examines if food, energy and nutrient intakes among individuals with self-reported 
food habit change were different compared with those of individuals with no food habit change. In order to be able better to interpret previous results, we investigated if the relationship between diet and breast cancer is influenced by factors that potentially are related to bias in selfreported dietary data: the study examined by stratified analysis if dietary change, obesity and misreporting of energy influence the association between selected dietary factors and breast cancer risk.

\section{Subjects and methods}

\section{Malmö Diet and Cancer cobort}

The MDC study is a population-based prospective cohort study in the third largest city of Sweden (250000 inhabitants). During the baseline examinations from March 1991 to October 1996, all men aged 50-73 years, and all women aged 45-73 years, and living in Malmo were invited to participate in the study. Only individuals with sufficient Swedish language skills were included in the study. With a participation rate of $\sim 40 \%$, the cohort consists of 28098 individuals that either joined the study spontaneously or joined after receiving a mail invitation. A more detailed description of the cohort is given elsewhere ${ }^{8,9}$.

Participants came to the study centre on two occasions. At the first visit, trained project staff provided groups of participants with information on the background and aims of the project, gave detailed instructions about the dietary data collection procedure, distributed the study questionnaire on lifestyle and medical history, etc. and the diet history questionnaire, and conducted direct anthropometrical measurements. At the second visit, individual interviews were conducted by trained dietary interviewers to complete the diet history (see description below) and to check the correctness of completed questionnaires.

\section{Study sample}

This study used an age-based definition of menopausal status. Natural menopause was determined in a group of women $(n=2898)$ in the MDC cohort without surgery and without hormone therapy. The median natural menopause age was 50.0 years. Therefore, only women with an age above 50 years were considered for this study $(n=12781)$. Three different versions of a socio-economic and lifestyle questionnaire were used during the baseline examinations. Only versions two and three had information on all variables examined in this project (creating a study population of 11429 women). Version two was used during February 1992 to December 1994 and version three was used from December 1994 to December 1996.

When analysing breast cancer relationships, all women above 50 years $(n=12781)$ (all versions of the questionnaire) were considered. The Swedish Cancer Registry and the Southern Swedish Regional Cancer Registry provided information on breast cancer cases from the time of the baseline examinations until the end of follow-up (31 December 2003). After excluding prevalent cancer cases, except cervical cancer in situ and nonmalignant skin carcinoma, a total of 11726 subjects remained. Out of these, a total of 428 incident breast cancer cases occurred during an average of 9.5 years of follow-up. Total person-years of follow-up were 110944 years.

\section{Past food babit change}

Past change in diet was derived from the questionnaire item 'Have you substantially changed your eating habits because of illness or some other reasons?' Subjects reporting a dietary change were asked for the reason for the food habit change in closed-ended answer categories covering both health-related and other reasons. The various reasons for change were aggregated into two categories. Category I (Cat I) included: hypertension, high blood lipids, overweight, diabetes, high blood sugar, coronary and other vascular diseases, and a wish to control body weight. The classification was based on the assumption that these reasons were associated with the metabolic syndrome and that these individuals might have made similar food habit changes because they would be given a similar type of dietary advice. Category II (Cat II) included reasons for change that were not associated with any obvious dietary pattern similarities: changed degree of physical activity, gastrointestinal disorder, mental disorder, allergy, changed working hours, changed work load, eat alone nowadays, unemployment, eat in company of others nowadays, worsened economy, retirement, disease in family and other reasons. A three-category variable was constructed discriminating among non-changers, diet changers with Cat I reasons and diet changers with Cat II reasons.

\section{Dietary metbodology}

Dietary data were collected through a modified diet history method, specifically developed for the MDC study. The method consists of a 7-day menu book that collected information on cooked lunches and dinners and cold beverages (including alcoholic beverages), and a 168-item questionnaire to obtain information on frequencies and portion sizes of regularly consumed foods during the past year. Diet assistants conducted a $1 \mathrm{~h}$ diet history interview with each participant, and carefully checked the menu book and questionnaire to make sure that the information provided did not overlap. The information on food intake was converted to nutrient intake data using the nutrient information available in the MDC study Food and Nutrient Database. This database, specifically developed for the MDC study, originates from PC KOST2-93 of the Swedish National Food Administration.

The validity of the diet history method has previously been examined with 18 days of weighed food records collected during 1 year as the reference ${ }^{10}$. In women, 
energy-adjusted correlations were for total fat 0.69 , fibre 0.69 , vegetables 0.53 and fruits 0.77 .

This study examined the following nutrient variables: energy (kcal), fibre $(\mathrm{g})$, total fat $(\mathrm{g})$, omega-6 fatty acids $(\mathrm{g})$, the percentage energy contribution ( $\mathrm{E} \%)$ of carbohydrate, protein, total fat, saturated fat, monounsaturated fat, polyunsaturated fat, and omega- 3 and omega- 6 polyunsaturated fatty acids.

The food groups examined in this study were: vegetables, fruits and berries, vegetable and fruit juice, high-fat meat, low-fat meat, sausages, eggs, high-fat fish, low-fat fish, boiled potatoes, fried and deep fried potatoes, rice and pasta, cereals, low-fibre bread, high-fibre bread, buns and cookies (biscuits), cheese, cottage cheese, highfat milk, low-fat milk, high-fat fermented milk, low-fat fermented milk, cream, ice cream, butter, low-fat margarine ( $40 \%$ fat), medium-fat margarine $(60-80 \%$ fat), other fats and oils, sweets and sugar, chocolates and snacks, coffee, tea, water, soft drinks, low-energy soft drinks, wine, beer and spirits. Fruit, berries and vegetables were also combined into one variable. The food group variables were expressed as the average amounts (g) consumed per day.

\section{Otber variables}

Information on age and gender was obtained through the person identification number.

A four-category variable was created for the four seasons of data collection: winter (December-February), spring (March-May), summer (June-August) and autumn (September-November). In September 1994, the coding of dietary data was slightly altered. Also 17 interviewers conducted the dietary history interviews during baseline examination. Therefore, analysis of dietary data was adjusted for season, method version (indicating data collection before or after 1 September 1994) and diet interviewer in order to control for undue influences of dietary data collection.

Other lifestyle and socio-economic variables were obtained through a standardised questionnaire. Education was categorised into five categories based on the type of education attained: elementary, primary and secondary, upper secondary, further education without a degree, and university degree. Smoking habits were categorised into current smoker (including irregular smoking), ex-smokers and non-smokers. Leisure-time physical activity was obtained from questions about different physical activities over the seasons where minutes per week of each activity were multiplied by an intensity factor creating a leisuretime physical activity score. The score was categorised into quartiles. Alcohol consumption was divided into four categories. Individuals with no consumption of alcohol in the menu book, and who indicated no consumption of alcohol during the previous year in the socio-economic and lifestyle questionnaire, were categorised as zero consumers. The other subjects were categorised according to an assumption of biological risk ${ }^{11} ;<15 \mathrm{~g}$ alcohol per day (low), 15-30 g (medium) and $>30 \mathrm{~g}$ (high).

Age at menarche was constructed from self-reported year of menarche and year of birth, and handled as a continuous variable. Current use of hormone replacement therapy (HRT) was based on information from both a questionnaire item of regular medication use and from the 7-day menu book, and dichotomised into yes/no. Parity was constructed from questions on the number of children, and aggregated into three answer categories: no children, one child and two or more children. Age at birth of the first child was constructed from the participant's year of birth, and the year of birth of the first child, and was divided into four categories: no children, $<24$ years, $24-30$ years and $>30$ years. Total duration of breast-feeding (cumulative for all child births) was divided into three categories (no breast-feeding, 1-6 months and $>6$ months of breast feeding). Dichotomous variables (yes/no) were constructed for miscarriage and ever use of contraceptive pills.

Anthropometric measurements, height, weight, and waist and hip circumference were taken by trained project staff and obtained from subjects without shoes and wearing light indoor clothing. The percentage body fat (BF\%) was determined through the body composition analyser system BIA 109 (JRL Systems), which uses the bioimpedance principle.

The physical activity level (PAL), expressed as energy expenditure divided by basal metabolic rate (BMR), is often used when identifying misreporters of energy, by comparing PAL with energy intake divided by BMR. Equations for predicting BMR from age, sex, weight and height recommended by the World Health Organization were used ${ }^{12}$ and total energy expenditure for each individual was calculated from self-reported information on physical activity at work, leisure-time physical activity and household work, and estimated sleeping hours, selfcare and passive time. This procedure is described in detail elsewhere $^{13}$. Hours per day of each activity were multiplied by an activity-specific factor creating individual PALs. Energy misreporting was defined as having a ratio of reported energy intake to BMR outside the $95 \%$ confidence interval (CI) limits of the calculated $\mathrm{PAL}^{13}$. A threecategory variable was constructed discriminating between low, adequate and high energy reporters.

\section{Statistical analysis}

The SPSS statistical computer package (version 11.5; SPSS Inc.) was used for all statistical analyses. The nominal level of $\alpha=0.05$ (i.e. $P<0.05$ ) was considered significant in two-sided tests. The continuous dietary variables were first log-transformed to normalise the distribution of data. Prior to log-transformation, a small amount $(0.01)$ was added to food variables to handle zero consumers.

First, a series of analyses were performed for food and nutrient variables, calculating geometric means and 
medians between past food habit change categories. Analysis of variance (using the general linear model procedure) examined differences in mean food and nutrient intakes between the two categories of dietary change and no dietary change. Multiple comparison tests were made with the least significant difference test, at $\alpha=0.05$. The analyses were adjusted for age, interview method version, season of interview and diet interviewer. A subsequent analysis also adjusted for total energy intake.

Differences in baseline characteristics between breast cancer cases and non-cases were examined with Student's $t$-test, Mann-Whitney $U$-test and $\chi^{2}$ analysis. The examined variables were: age at baseline, height, weight, body mass index (BMI), waist circumference, hip circumference, education, leisure-time physical activity, alcohol habits, smoking habits, current use of HRT, ever use of contraceptive pills, age at birth of first child, miscarriage, parity and breast-feeding.

Total fat, omega-6 fatty acids, fibre, and 'fruit, berries and vegetables' were categorised into quintiles based on the residuals obtained when regressing the specific variable on total energy intake ${ }^{1}$. The median intakes of these variables were identified for each quintile.

We estimated incidence rate ratios (IRRs) with 95\% CI using Cox proportional hazard regression to examine the associations between intakes of total fat, omega- 6 fatty acids, fibre and 'fruit, berries and vegetables', and breast cancer incidence, with the first quintile of each exposure type as the reference. The basic model included age, method version, diet interviewer, season and total energy intake as covariates. The second model was extended to include risk factors and potential confounding variables: height, weight, education, current use of HRT, use of contraceptive pills, age at birth of first child, alcohol consumption and leisure-time physical activity. These variables have in previous analyses been identified as potential confounders in the MDC data $\operatorname{set}^{14,15}$. Analyses were repeated to examine trends across quintiles. Tests of interaction were performed for the dietary categories and dietary change categories. Quintiles of fibre and omega-6 fatty acids, respectively, and dietary change status were cross-classified and the breast cancer risk computed for each cell, with the lowest quintile among subjects without dietary change as the reference category. Moreover, Cox regression analyses estimated the association between intakes of total fat, fibre, omega- 6 fatty acids and 'fruit, berries and vegetables' and risk of breast cancer in separate strata of low, adequate and high energy reporters. Similarly, we also estimated the breast cancer risk for the dietary variables within each stratum of obesity status (BMI $\leq 27 \mathrm{~kg} \mathrm{~m}^{-2}$ and $>27 \mathrm{~kg} \mathrm{~m}^{-2}$ ).

\section{Results}

Higher intakes of fruit, berries and vegetables, high-fibre bread, low-fat milk and low-fat fermented milk, and lower intake of low-fibre bread, whole milk and whole fermented milk were observed among individuals with the Cat I reasons for the diet change compared with the other two groups of individuals (Table 1). Cat I also consumed less sweets, snacks and soft drinks, and more low-fat margarine. Non-changers reported a higher intake of boiled potatoes, cookies, cheese, cream and ice cream, medium-fat margarine, coffee, wine and beer than individuals with dietary change. A lower consumption of meat, sausages, low-fat fish and coffee was observed among Cat II, but they reported higher tea consumption. When analysing energyadjusted food variables, the consumption of low-fibre bread, chocolates and soft drinks between the two types of reasons for change was not significantly different (data not shown). Individuals of Cat I had lower energy intakes and lower percentage energy from fat, especially saturated fat. Higher intakes of fibre were observed among individuals overall with dietary change compared with individuals reporting stable food habits.

Breast cancer cases were younger than non-cases at baseline. Cases were also taller and heavier. They were more often users of current HRT and ever users of contraceptive pills (Table 2).

\section{Past food babit change}

The risk of breast cancer incidence appears elevated in individuals reporting having had a food habit change in the past compared with individuals not reporting a food habit change. However, the relationship is not significant $(\mathrm{IRR}=1.14 ; 95 \% \mathrm{CI}=0.92-1.40)$.

The risk of breast cancer incidence was examined across quintiles of fibre, total fat, omega- 6 fatty acids and 'fruit, berries and vegetables' intakes (Table 3 ). When examining all individuals, none of the quintiles of total fat intake was significantly associated with breast cancer risk with the first quintile as the reference, but a borderline trend $(P=0.06)$ was observed across the quintiles when adjusting for potential confounders. Similarly, when analysing separate strata of food habit change status, a borderline trend of increased breast cancer risk across quintiles of fat was observed among non-diet changers, but this trend was not seen among diet changers.

For omega- 6 fatty acids, a positive trend across quintiles was observed when examining all individuals (as expected from previous results ${ }^{15}$ ); this relationship did not change when including potential confounders in the analysis. The trend was stronger in individuals with no reported dietary change. In changers, the third compared with the first quintile showed a decreased breast cancer risk.

Fibre intakes were not significantly related to breast cancer risk when analysing all individuals. However, quintiles two and five showed a significantly decreased breast cancer risk among individuals without reported dietary change, but there was no significant trend across quintiles. When adjusting for potential confounders, only quintile two showed a significantly decreased risk (data 
Table 1 Food group and nutrient intakes among women with different reasons for food habit change and women without food habit change in a sample $(n=11429)$ from the Malmö Diet and Cancer cohort 1991-1996

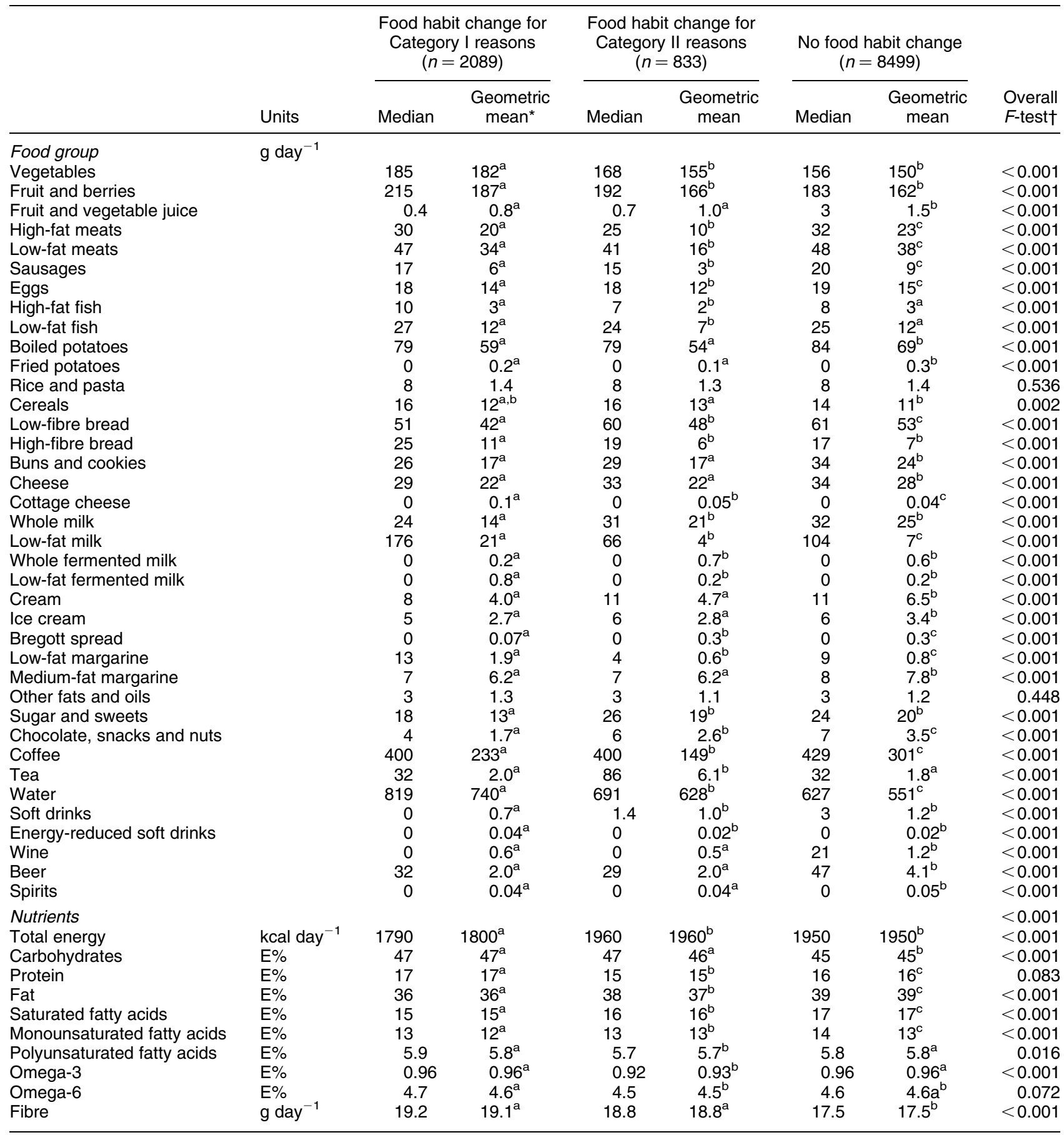

E\% - percentage of energy.

${ }^{*}$ Adjusted for season of diet interview, diet assistant, method version and age at baseline examination.

$\dagger$ Test of overall mean differences.

${ }_{\mathrm{a}, \mathrm{b}}$ Dissimilar letters indicate significant differences of the mean intakes in the multiple comparisons test (least squares difference) at $\alpha=0.05$.

not shown). Among changers, the second quintile showed an increased risk.

The third quintile of 'fruit, berries and vegetables' was associated with decreased breast cancer risk, when all individuals were included in the analysis. Among individuals reporting stable dietary habits, a borderline significant decrease in breast cancer risk was observed across quintiles of 'fruit, berries and vegetables', with the third and highest quintile of intake associated with decreased breast cancer risk. Adjusting for potential confounders did not influence the associations; however, the trend across quintiles became weaker. This trend 
Table 2 Baseline characteristics of cases and non-cases of post-menopausal women from the Malmö Diet and Cancer cohort, 1991-2003

\begin{tabular}{|c|c|c|c|}
\hline & Cases $(n=428)$ & Non-cases $(n=11726)$ & $P$-value \\
\hline & & Mean (SD) & \\
\hline Age (years) & $59.4(6.0)$ & $60.4(6.5)$ & $<0.01$ \\
\hline Height $(\mathrm{cm})$ & $163.9(5.7)$ & $163.1(6.0)$ & $<0.01$ \\
\hline Weight (kg) & $69.6(11.9)$ & $68.2(11.8)$ & 0.02 \\
\hline Waist circumference (cm) & $79.2(10.7)$ & $78.5(10.7)$ & 0.25 \\
\hline Hip circumference $(\mathrm{cm})$ & $99.2(9.9)$ & $98.6(9.7)$ & 0.20 \\
\hline Body mass index $\left(\mathrm{kg} \mathrm{m}^{-2}\right)$ & 25.9 (4.3) & $25.7(4.3)$ & 0.30 \\
\hline \multirow[t]{2}{*}{ Percentage body fat } & $31.6(4.8)$ & $31.3(4.8)$ & 0.32 \\
\hline & & Frequency (\%) & \\
\hline \multicolumn{4}{|l|}{ Educational status } \\
\hline Elementary & $180(42)$ & $5153(46)$ & \\
\hline Primary and secondary & 140 (33) & $3428(30)$ & \\
\hline Upper secondary & $22(5)$ & $626(6)$ & \\
\hline Further education without degree & $27(6)$ & $835(7)$ & \\
\hline University degree & $57(13)$ & $1223(11)$ & 0.17 \\
\hline \multicolumn{4}{|l|}{ Leisure-time physical activity } \\
\hline Quintile 1 & $73(17)$ & $2256(20)$ & \\
\hline Quintile 2 & $102(24)$ & $2223(20)$ & \\
\hline Quintile 3 & $84(20)$ & $2229(20)$ & \\
\hline Quintile 4 & $95(22)$ & $2240(20)$ & \\
\hline Quintile 5 & $71(17)$ & $2272(20)$ & 0.69 \\
\hline \multicolumn{4}{|l|}{ Alcohol consumption } \\
\hline Non-drinkers & $31(7)$ & $995(9)$ & \\
\hline Low $(<15 \mathrm{~g})$ & $332(78)$ & $8693(77)$ & \\
\hline Medium (15-30 g) & $49(11)$ & 1395 (12) & \\
\hline $\operatorname{High}(>30 \mathrm{~g})$ & $16(4)$ & $215(2)$ & 0.24 \\
\hline \multicolumn{4}{|l|}{ Smoking habits } \\
\hline Current smokers & $110(26)$ & $2931(26)$ & \\
\hline Ex-smokers & $131(31)$ & $2986(26)$ & \\
\hline Non-smokers & $187(44)$ & $5376(48)$ & 0.13 \\
\hline \multicolumn{4}{|l|}{ Current hormone replacement therapy } \\
\hline Yes & $136(35)$ & $2243(21)$ & \\
\hline No & $257(65)$ & $8377(79)$ & $<0.01$ \\
\hline \multicolumn{4}{|l|}{ Use of contraceptive pills } \\
\hline Yes & $192(45)$ & $4521(40)$ & \\
\hline No & $235(55)$ & $6767(60)$ & 0.04 \\
\hline \multicolumn{4}{|l|}{ Miscarriage } \\
\hline Yes & $84(20)$ & $2450(22)$ & \\
\hline No & 339 (80) & 8768 (78) & 0.33 \\
\hline \multicolumn{4}{|l|}{ Parity } \\
\hline No children & $53(13)$ & $1392(13)$ & \\
\hline One child & $86(21)$ & $2415(22)$ & \\
\hline Two or more children & $274(66)$ & 7296 (66) & 0.85 \\
\hline \multicolumn{4}{|l|}{ Age at birth of first child } \\
\hline$<24$ years & $141(33)$ & 4202 (37) & \\
\hline $24-29.9$ years & $164(38)$ & $4108(36)$ & \\
\hline$\geq 30$ years & $55(13)$ & $1390(12)$ & \\
\hline No children & $68(16)$ & $1598(14)$ & 0.33 \\
\hline \multicolumn{4}{|l|}{ Age at menarche } \\
\hline$<13$ years & $187(44)$ & $4987(45)$ & \\
\hline$>13$ years & $234(56)$ & $6216(56)$ & 0.97 \\
\hline \multicolumn{4}{|l|}{ Breast-feeding } \\
\hline 0 months & $106(25)$ & $2436(22)$ & \\
\hline $1-6$ months & 126 (29) & 3553 (31) & \\
\hline 7 months or more & $196(46)$ & $5309(47)$ & 0.32 \\
\hline
\end{tabular}

SD - standard deviation.

${ }^{*} t$-test was used to compare means; $x^{2}$ analysis to compare frequency distributions of smoking habits, current hormone replacement therapy, use of contraceptive pills, miscarriage and age at menarche, and Mann-Whitney U-test to compare the ranking of educational status, leisure-time physical activity, alcohol consumption, parity and breast-feeding.

across 'fruit, berries and vegetables' quintiles was not observed among diet changers.

The tests of interaction were significant between omega-6 fatty acids and dietary change categories $(P=0.004)$, and between fibre and dietary change categories $(P=0.003)$. The interaction was still significant for omega- 6 intake when examined as a continuous variable $(P=0.04)$, but not for fibre $(P=0.94)$. When omega- 6 fatty acid quintiles were cross-classified with dietary change status, both low and high intakes of omega- 6 fatty acids showed increased breast cancer risks among diet changers when compared with the first 
Table 3 Incidence rate ratios (IRRs) of postmenopausal breast cancer in separate strata of food habit change status in the Malmö Diet and Cancer cohort $1991-2003^{\star}$

\begin{tabular}{|c|c|c|c|c|c|c|c|c|c|c|}
\hline \multirow{2}{*}{$\begin{array}{l}\text { Quintiles of } \\
\text { dietary intake }\end{array}$} & \multirow{2}{*}{$\begin{array}{l}\text { Median intake } \\
\quad\left(\mathrm{g} \mathrm{day}^{-1}\right)\end{array}$} & \multicolumn{3}{|c|}{ All subjects } & \multicolumn{3}{|c|}{ No dietary change } & \multicolumn{3}{|c|}{ Dietary change } \\
\hline & & Person-years/cases & IRR & $95 \% \mathrm{Cl}$ & Person-years/cases & IRR & $95 \% \mathrm{Cl}$ & Person-years/cases & IRR & $95 \% \mathrm{Cl}$ \\
\hline \multicolumn{11}{|l|}{ Fat } \\
\hline 1 & 62.5 & $22497 / 80$ & 1.00 & - & $13684 / 47$ & 1.00 & - & $8771 / 33$ & 1.00 & - \\
\hline 2 & 74.7 & $22454 / 76$ & 0.95 & $0.69-1.30$ & $15722 / 49$ & 0.91 & $0.61-1.36$ & $6713 / 27$ & 1.04 & $0.62-1.72$ \\
\hline 3 & 81.4 & $22033 / 97$ & 1.26 & $0.94-1.70$ & $17033 / 69$ & 1.20 & $0.83-1.74$ & $5000 / 28$ & 1.48 & $0.89-2.46$ \\
\hline 4 & 86.3 & $22087 / 83$ & 1.07 & $0.79-1.46$ & $17582 / 67$ & 1.14 & $0.78-1.65$ & $4473 / 16$ & 0.94 & $0.52-1.72$ \\
\hline 5 & 96.1 & $21873 / 92$ & 1.21 & $\begin{array}{c}0.90-1.64 \\
P \text { for trend } 0.14\end{array}$ & $17577 / 72$ & 1.22 & $\begin{array}{c}0.85-1.77 \\
P \text { for trend } 0.14\end{array}$ & $4275 / 20$ & 1.25 & $\begin{array}{c}0.72-2.19 \\
P \text { for trend } 0.48\end{array}$ \\
\hline \multicolumn{11}{|c|}{ Omega- 6 fatty acids } \\
\hline 1 & 6.6 & $21534 / 65$ & 1.00 & - & $15850 / 38$ & 1.00 & - & $5665 / 27$ & 1.00 & - \\
\hline 2 & 8.5 & $21830 / 82$ & 1.24 & $0.90-1.72$ & $16154 / 57$ & 1.48 & $0.98-2.23$ & $5649 / 25$ & 0.85 & $0.49-1.47$ \\
\hline 3 & 9.7 & $22463 / 78$ & 1.15 & $0.83-1.60$ & $16405 / 65$ & 1.65 & $1.10-2.46$ & $6035 / 13$ & 0.40 & $0.20-0.77$ \\
\hline 4 & 11.2 & $22722 / 99$ & 1.44 & $1.05-1.97$ & $16772 / 62$ & 1.55 & $1.03-2.32$ & $5930 / 37$ & 1.17 & $0.71-1.93$ \\
\hline 5 & 14.1 & $22395 / 104$ & 1.54 & $\begin{array}{c}1.13-2.10 \\
P \text { for trend } 0.004\end{array}$ & $16417 / 82$ & 2.09 & $\begin{array}{c}1.42-3.08 \\
P \text { for trend } 0.0005\end{array}$ & $5952 / 22$ & 0.69 & $\begin{array}{c}0.39-1.22 \\
P \text { for trend } 0.64\end{array}$ \\
\hline \multicolumn{11}{|l|}{ Fibre } \\
\hline 1 & 12.5 & $21291 / 89$ & 1.00 & - & $17736 / 79$ & 1.00 & - & $3536 / 10$ & 1.00 & - \\
\hline 2 & 16.0 & $21900 / 74$ & 0.80 & $0.59-1.09$ & $17924 / 47$ & 0.58 & $0.41-0.84$ & $3958 / 27$ & 2.25 & $1.08-4.66$ \\
\hline 3 & 18.3 & $22009 / 82$ & 0.88 & $0.65-1.18$ & $16875 / 64$ & 0.85 & $0.61-1.18$ & $5111 / 18$ & 1.15 & $0.53-2.49$ \\
\hline 4 & 21.0 & $22497 / 104$ & 1.05 & $0.79-1.40$ & $15728 / 74$ & 1.02 & $0.74-1.41$ & $6747 / 30$ & 1.41 & $0.69-2.90$ \\
\hline 5 & 25.9 & $23247 / 79$ & 0.77 & $\begin{array}{c}0.57-1.05 \\
P \text { for trend } 0.51\end{array}$ & $13334 / 40$ & 0.63 & $\begin{array}{c}0.43-0.93 \\
P \text { for trend } 0.45\end{array}$ & $9879 / 39$ & 1.26 & $\begin{array}{c}0.63-2.55 \\
P \text { for trend } 0.54\end{array}$ \\
\hline \multicolumn{11}{|c|}{ Fruit, berries and vegetables } \\
\hline 1 & 190 & $21291 / 94$ & 1.00 & - & $17302 / 79$ & 1.00 & - & $3960 / 15$ & 1.00 & - \\
\hline 2 & 291 & $21849 / 80$ & 0.83 & $0.61-1.11$ & $17032 / 61$ & 0.79 & $0.56-1.10$ & $4807 / 19$ & 0.95 & $0.48-1.87$ \\
\hline 3 & 371 & $22063 / 69$ & 0.70 & $0.51-0.95$ & $16894 / 50$ & 0.63 & $0.44-0.91$ & $5157 / 19$ & 0.93 & $0.47-1.84$ \\
\hline 4 & 464 & $22810 / 102$ & 0.99 & $0.74-1.31$ & $16387 / 73$ & 0.94 & $0.68-1.30$ & $6405 / 29$ & 1.15 & $0.61-2.15$ \\
\hline 5 & 626 & $22932 / 83$ & 0.78 & $\begin{array}{c}0.57-1.05 \\
P \text { for trend } 0.35\end{array}$ & $13983 / 41$ & 0.59 & $\begin{array}{c}0.40-0.87 \\
P \text { for trend } 0.052\end{array}$ & $8903 / 42$ & 1.13 & $\begin{array}{c}0.62-2.06 \\
P \text { for trend } 0.45\end{array}$ \\
\hline
\end{tabular}

$\mathrm{Cl}-$ confidence interval.

*Adjusted for total energy, age, method version, diet interviewer and season. 
quintile among non-changers, suggesting that the background risk of women with dietary change was elevated (Fig. 1). When fibre quintiles were cross-classified with dietary change status, none of the risk estimates for fibre were significant among changers.

\section{Energy reporting}

When analysing only adequate energy reporters, the results were similar compared with analysing all individuals. Therefore, excluding individuals reporting either low or high energy intake did not change the risk estimates for any of the analysed food variables (data not shown).

\section{Obesity}

The last set of analyses compared risk estimates across food and nutrient quintiles within obesity status categories (Table 4). Among individuals with a BMI below $27 \mathrm{~kg} \mathrm{~m}^{-2}$, high intakes of total fat were, with a significant positive trend, associated with increased breast cancer risk. Similarly, the fourth and fifth quintiles of omega- 6 fatty acids were associated with an increased risk in this stratum, and the positive trend was clearly significant. Decreased risks were observed in the second and fifth quintile of fibre intake among individuals with a BMI below $27 \mathrm{~kg} \mathrm{~m}^{-2}$, but no significant trend across quintiles was observed. A significant decreased risk of high intakes of 'fruit, berries and vegetables' with a significant trend was also seen among these individuals. In contrast, no significant relationships were observed with these dietary variables among women with a BMI above $27 \mathrm{~kg} \mathrm{~m}^{-2}$.

\section{Discussion}

Individuals reporting past food habit change due to health reasons related to the metabolic syndrome (Cat I) had higher intakes of fruits, vegetables, high-fibre bread, lowfat milk and fermented milk compared with non-changers and changers for Cat II reasons. Many of these food groups are recognised as healthier foods, and are often recommended to people with obesity and health disorders associated with the metabolic syndrome. Cat I diet changers also had a lower reported total energy intake.

When past food habit changers, who can be particularly prone to errors in dietary self-reports, were excluded from the analysis, the observed association between omega- 6 fatty acids and 'fruit, berries and vegetables' and postmenopausal breast cancer was stronger, while associations with total fat and fibre intakes remained almost unchanged compared with analysing all women. Stratifying on obesity status had an impact on risk estimates for all food variables examined; more pronounced risk estimates among individuals with BMI below $27 \mathrm{~kg} \mathrm{~m}^{-2}$. Excluding individuals reporting non-adequate energy intakes did not influence the interpretation of results of any of the energy-adjusted food and nutrient variables.

This study may help the interpretation of many previous epidemiological studies that have produced null results, in spite of animal and ecological studies indicating strong dietary associations. Cancer development is believed to involve long latency periods. Individuals who report past food habit change might therefore both report and consume diets that are different from those responsible for the development of tumours. Thus the reason for not observing any consistent relationship between diet and breast cancer among diet changers (in this study) may be due to unstable diets, and consequently misclassification among dietary exposure categories in relation to tumour development. However, the dietary change variable in this study is very crude, potentially associated with many

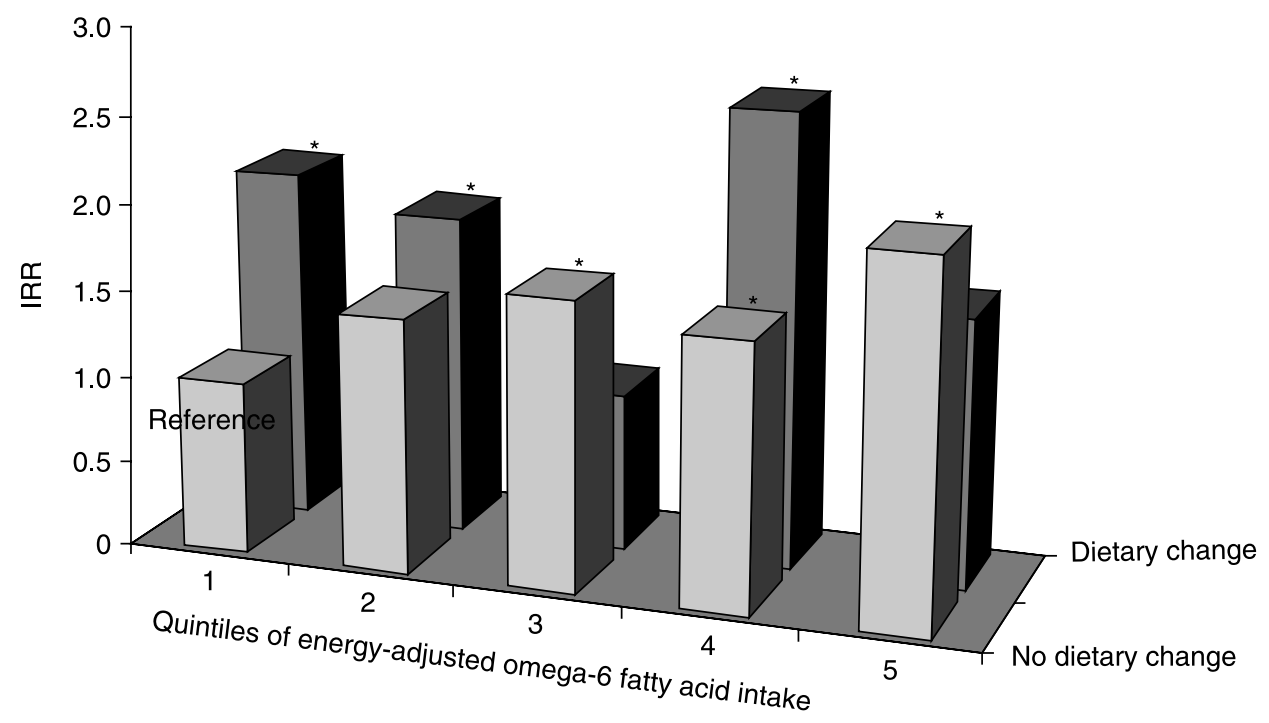

Fig. 1 Incident rate ratios (IRRs) of postmenopausal breast cancer after cross-classification of omega- 6 fatty acids against dietary change status. Adjusted for age, method version, diet assistant and season. ${ }^{*} P<0.05$, in Cox regression with the first quintile of those with no dietary change as the reference category 
Table 4 Incidence rate ratios (IRRs) of postmenopausal breast cancer in separate strata of BMI status in the Malmö Diet and Cancer Cohort*

\begin{tabular}{|c|c|c|c|c|c|c|}
\hline \multirow{2}{*}{$\begin{array}{l}\text { Quintiles of } \\
\text { dietary intake }\end{array}$} & \multicolumn{3}{|c|}{$\mathrm{BMI}<27 \mathrm{~kg} \mathrm{~m}^{-2}$} & \multicolumn{3}{|c|}{$\mathrm{BMI}>27 \mathrm{~kg} \mathrm{~m}^{-2}$} \\
\hline & Person-years/cases & IRR & $95 \% \mathrm{Cl}$ & Person-years/cases & IRR & $95 \% \mathrm{Cl}$ \\
\hline \multicolumn{7}{|l|}{ Fat } \\
\hline 1 & $15020 / 45$ & 1.00 & - & $7377 / 35$ & 1.00 & - \\
\hline 2 & $15422 / 47$ & 1.01 & $0.67-1.52$ & $6919 / 29$ & 0.92 & $0.56-1.51$ \\
\hline 3 & $15150 / 64$ & 1.43 & $0.97-2.09$ & $6825 / 33$ & 1.07 & $0.67-1.74$ \\
\hline 4 & $15264 / 50$ & 1.11 & $0.74-1.66$ & $6731 / 33$ & 1.09 & $0.67-1.76$ \\
\hline 5 & $15071 / 70$ & 1.59 & $\begin{array}{c}1.09-2.32 \\
P \text { for trend } 0.015\end{array}$ & $6713 / 22$ & 0.72 & $\begin{array}{c}0.42-1.23 \\
P \text { for trend } 0.48\end{array}$ \\
\hline \multicolumn{7}{|c|}{ Omega- 6 fatty acids } \\
\hline 1 & $15432 / 40$ & 1.00 & - & $6029 / 25$ & 1.00 & - \\
\hline 2 & $15119 / 54$ & 1.37 & $0.91-2.06$ & $6624 / 28$ & 1.02 & $0.59-1.75$ \\
\hline 3 & $15127 / 48$ & 1.22 & $0.80-1.86$ & $7280 / 30$ & 1.00 & $0.59-1.71$ \\
\hline 4 & $15463 / 63$ & 1.55 & $1.04-2.31$ & $7132 / 36$ & 1.23 & $0.74-2.06$ \\
\hline 5 & $14786 / 71$ & 1.84 & $\begin{array}{c}1.24-2.71 \\
P \text { for trend } 0.002\end{array}$ & $7499 / 33$ & 1.07 & $\begin{array}{c}0.63-1.80 \\
P \text { for trend } 0.59\end{array}$ \\
\hline \multicolumn{7}{|l|}{ Fibre } \\
\hline 1 & $14662 / 63$ & 1.00 & - & $6557 / 26$ & 1.00 & - \\
\hline 2 & $14843 / 43$ & 0.67 & $0.46-0.99$ & $6968 / 31$ & 1.08 & $0.64-1.82$ \\
\hline 3 & $15061 / 59$ & 0.89 & $0.62-1.27$ & $6865 / 23$ & 0.83 & $0.47-1.46$ \\
\hline 4 & $15394 / 70$ & 1.01 & $0.71-1.42$ & $7010 / 34$ & 1.16 & $0.69-1.95$ \\
\hline 5 & $15966 / 41$ & 0.57 & $\begin{array}{c}0.38-0.85 \\
P \text { for trend } 0.13\end{array}$ & $7164 / 38$ & 1.23 & $\begin{array}{c}0.74-2.05 \\
P \text { for trend } 0.37\end{array}$ \\
\hline \multicolumn{7}{|c|}{ Fruit, berries and vegetables } \\
\hline 1 & $14801 / 66$ & 1.00 & - & $6410 / 28$ & 1.00 & - \\
\hline 2 & $15078 / 57$ & 0.85 & $0.59-1.21$ & $6692 / 23$ & 0.80 & $0.46-1.39$ \\
\hline 3 & $15182 / 49$ & 0.72 & $0.50-1.04$ & $6782 / 20$ & 0.66 & $0.37-1.17$ \\
\hline 4 & $15540 / 56$ & 0.78 & $0.55-1.12$ & $7115 / 46$ & 1.46 & $0.91-2.36$ \\
\hline 5 & $15325 / 48$ & 0.66 & $\begin{array}{c}0.46-0.97 \\
P \text { for trend } 0.035\end{array}$ & $7565 / 35$ & 0.97 & $\begin{array}{c}0.58-1.62 \\
P \text { for trend } 0.30\end{array}$ \\
\hline
\end{tabular}

$\mathrm{BMI}$ - body mass index; $\mathrm{Cl}$ - confidence interval.

${ }^{*}$ Adjusted for age, method version, diet assistant and season.

confounding factors, and prone to errors. For instance, changers are more obese than non-changers (as previously shown with these data $)^{5}$, and obesity is associated with postmenopausal breast cancer in many studies ${ }^{16}$. It is likely that pathological conditions (such as type 2 diabetes) are more common in these women because of obesity, and therefore they possibly have different risk profiles in relation to breast cancer than non-changers of diet. For example, postmenopausal obese women have higher sex hormone levels compared with lean women ${ }^{17}$, and studies have shown higher prevalence of type 2 diabetes, hypertension and dyslipidaemia among breast cancer cases compared with women with benign breast pathology and controls ${ }^{18}$. Previous dietary change may also result in weight change. Although weight loss has been associated with increased mortality and coronary morbidity ${ }^{19}$, weight loss during adult life has instead been associated with decreased risk of postmenopausal breast cancer ${ }^{20}$. Thus the risk profile of diet changes may be quite complex.

This study could only examine self-reported food habit change, which is a limitation. Since we are not able to establish the degree of dietary change, the past food habit change variable is difficult to interpret. Studies analysing dietary factors in the MDC study have, so far, adjusted for past food habit change. Adjustment may, however, be incorrect because dietary factors may show different relationships with past food habit change, as indicated by the significant interaction tests in this study. Indeed, the literature indicates that adjustments for suspected errors (when the assumptions are wrong) can make matters worse $21-23$

Among diet changers, there was a higher percentage of those reporting low energy intakes (data not shown). Only minor changes were seen, however, in the associations between the examined dietary factors and risk of breast cancer when non-adequate energy reporters were excluded. Previous observations in the MDC study, investigating the influence of excluding those with inadequate energy reports, showed that the risk associated with alcohol increased and became significant. Alcohol intake categories in that study were based on absolute intake levels, while the other food and nutrient categories were based on energy-adjusted intakes (residual method). One goal of energy adjustment in nutrition epidemiological studies is to reduce the influence of reporting errors $^{24}$. Thus energy adjustment could explain why the results were similar when misreporters of energy were excluded from the analyses presented in this report.

The higher proportion of obese individuals among diet changers $^{5}$ may indicate a tendency to restrict intake . $^{6}$ Black $^{25}$ described under-reporting among post-obese individuals and suggested it to be related to dieting and 
dietary restraint, i.e. the tendency consciously to control food intake in order to assist weight loss or prevent weight gain $^{6}$. The dietary habits before a lifestyle change may be positively related to body fatness and to the development of the disease, but dietary habits after the change may have a different biological relationship. This study indicates different risk trends depending on obesity status, suggesting that obesity status and stratified analysis should be considered in dietary studies.

Although few studies have focused on the fact that dietary habits change over time, others have examined the impact of dieting behaviours ${ }^{26}$. For instance, inverse associations between BMI and the reported energy intake have been reported to be reduced by $\sim 20 \%$ when controlling for low energy dieting ${ }^{27}$. Studies have also used more sophisticated approaches and examined social desirability and social approval, or restraining behaviours concurrent with the assessment of diet. For instance, Hebert et al. have reported that important factors in predicting under-reporting of energy intake include dissatisfaction with body image and percentage body fat ${ }^{28,29}$. Doubly labelled water has enabled confirmation of under-reporting biases to be associated with both social desirability and education in women ${ }^{28}$. Such information is of specific interest because researchers need to identify groups of individuals that possibly report data of lower validity ${ }^{30}$, and sometimes specific information can be used in modelling of data or direct adjustment of nutrient intake values ${ }^{31}$.

Few studies have investigated the clinical implications of under-reporting. Rosell et al. investigated the problem of misreporting on the association between dietary factors and metabolic syndrome. Under-reporters had a higher prevalence of the metabolic syndrome (greater waist circumference and higher systolic and diastolic blood pressures) than did the non-under-reporters. The associations between fasting insulin concentrations and the intakes of polyunsaturated fats, omega- 6 fats and fat from milk products were stronger in under-reporters than in non-under-reporters, which indicates that inaccurate data can introduce spurious associations ${ }^{32}$. Macdiarmid et al. assessed the relationship of dietary fat and sugar consumption in men and women with different BMI. In the case of the women, the inclusion of low energy reporters completely reversed the relationship between consumption of high-fat, sweet foods and BMI due to reduced reporting of these products by obese women ${ }^{33}$.

We conclude that epidemiological studies should consider examining separate strata of dietary confounders (such as obesity and food habit change status) to help in interpreting results regarding diet-disease associations.

\section{Acknowledgements}

Sources of funding: Supported by the Swedish Cancer Society, the Swedish Medical Research Council, the European Commission and the City of Malmö.
Conflict of interest declaration: None of the authors had any conflicts of interest.

Authorship responsibilities: E.S., E.W. and B.G. contributed to study design. E.S. conducted the analysis of data, and wrote most of the manuscript with the assistance of E.W. and B.G. All authors reviewed the final manuscript.

\section{References}

1 Willett W. Nutritional Epidemiology. Oxford: Oxford University Press, 1998.

2 Tarasuk VS, Brooker AS. Interpreting epidemiologic studies of diet-disease relationships. Journal of Nutrition 1997; 127: $1847-52$.

3 Margetts BM, Nelson M. Overview of the principles of nutritional epidemiology. In: Margetts BM, Nelson M, eds. Design Concepts in Nutritional Epidemiology, 2nd ed. Oxford: Oxford University Press, 1997; xv, 451.

4 Wirfalt E, Mattisson I, Gullberg B, Berglund G. Food patterns defined by cluster analysis and their utility as dietary exposure variables: a report from the Malmo Diet and Cancer Study. Public Health Nutrition 2000; 3: $159-73$.

5 Sonestedt E, Wirfalt E, Gullberg B, Berglund G. Past food habit change is related to obesity, lifestyle and socioeconomic factors in the Malmo Diet and Cancer Cohort. Public Health Nutrition 2005; 8: 876-85.

6 Hill RJ, Davies PS. The validity of self-reported energy intake as determined using the doubly labelled water technique. British Journal of Nutrition 2001; 85: 415-30.

7 Lissner L. Measuring food intake in studies of obesity. Public Health Nutrition 2002; 5: 889-92.

8 Berglund G, Elmstahl S, Janzon L, Larsson SA. The Malmo Diet and Cancer Study. Design and feasibility. Journal of Internal Medicine 1993; 233: 45-51.

9 Manjer J, Carlsson S, Elmstahl S, Gulberg B, Janzon L, Lindstrom M, et al. The Malmo Diet and Cancer Study: representativity, cancer incidence and mortality in participants and non-participants. European Journal of Cancer Prevention 2001; 10: 489-99.

10 Elmstahl S, Riboli E, Lindgarde F, Gullberg B, Saracci R. The Malmo Food Study: the relative validity of a modified diet history method and an extensive food frequency questionnaire for measuring food intake. European Journal of Clinical Nutrition 1996; 50: 143-51.

11 Royal College of Psychiatrists. Alcohol: Our Favourite Drug: New Report on Alcohol and Alcohol-related Problems from a Special Committee of the Royal College of Psychiatrists. London: Tavistock, 1986.

12 Food and Agriculture Organization (FAO)/World Health Organization (WHO)/United Nations University (FAO/WHO/UNU). Energy and Protein Requirements: Report of a Joint FAO/WHO/UNU Expert Consultation. Geneva: WHO, 1985.

13 Mattisson I, Wirfalt E, Aronsson CA, Wallstrom P, Sonestedt E, Gullberg B, et al. Misreporting of energy: prevalence, characteristics of misreporters and influence on observed risk estimates in the Malmo Diet and Cancer cohort. British Journal of Nutrition 2005; 94: 832-42.

14 Mattisson I, Wirfalt E, Wallstrom P, Gullberg B, Olsson H, Berglund G. High fat and alcohol intakes are risk factors of postmenopausal breast cancer: a prospective study from the Malmo diet and cancer cohort. International Journal of Cancer 2004; 110: 589-97.

15 Wirfalt E, Mattisson I, Gullberg B, Johansson V, Olsson H, Berglund G. Postmenopausal breast cancer is associated with high intakes of omega6 fatty acids (Sweden). Cancer Causes \& Control 2002; 13: 883-93. 
16 Lahmann PH, Lissner L, Gullberg B, Olsson H, Berglund G. A prospective study of adiposity and postmenopausal breast cancer risk: the Malmo Diet and Cancer Study. International Journal of Cancer 2003; 103: 246-52.

17 Potischman N, Swanson CA, Siiteri P, Hoover RN. Reversal of relation between body mass and endogenous estrogen concentrations with menopausal status. Journal of the National Cancer Institute 1996; 88: 756-8.

18 Sinagra D, Amato C, Scarpilta AM, Brigandi M, Amato M, Saura G, et al. Metabolic syndrome and breast cancer risk. European Review of Medical and Pharmacological Science 2002; 6: 55-9.

19 Lahmann PH, Lissner L, Gullberg B, Berglund G. A prospective study of adiposity and all-cause mortality: the Malmo Diet and Cancer Study. Obesity Research 2002; 10: $361-9$.

20 Trentham-Dietz A, Newcomb PA, Egan KM, Titus-Ernstoff L, Baron JA, Storer BE, et al. Weight change and risk of postmenopausal breast cancer (United States). Cancer Causes \& Control 2000; 11: 533-42.

21 Flegal KM, Brownie C, Haas JD. The effects of exposure misclassification on estimates of relative risk. American Journal of Epidemiology 1986; 123: 736-51.

22 Flegal KM, Keyl PM, Nieto FJ. Differential misclassification arising from nondifferential errors in exposure measurement. American Journal of Epidemiology 1991; 134: 1233-44.

23 Plummer M, Clayton D. Measurement error in dietary assessment: an investigation using covariance structure models. Part II. Statistics in Medicine 1993; 12: 937-48.

24 Willett WC, Howe GR, Kushi LH. Adjustment for total energy intake in epidemiologic studies. American Journal of Clinical Nutrtion 1997; 65: 1220S-8S; discussion 29S-31S.

25 Black AE, Jebb SA, Bingham SA, Runswick SA, Poppitt SD. The validation of energy and protein intakes by double labeled water and 24-hour urinary nitrogen excretion in post-obese subjects. Journal of Human Nutrition and Dietetics 1995; 8: 51-64.
26 Neumark-Sztainer D, Jeffery RW, French SA. Self-reported dieting: how should we ask? What does it mean? Associations between dieting and reported energy intake. International Journal of Eating Disorders 1997; 22: 437-49.

27 Ballard-Barbash R, Graubard I, Krebs-Smith SM, Schatzkin A, Thompson FE. Contribution of dieting to the inverse association between energy intake and body mass index. European Journal of Clinical Nutrtion 1996; 50: 98-106.

28 Hebert JR, Ebbeling CB, Matthews CE, Hurley TG, Ma Y, Druker S, et al. Systematic errors in middle-aged women's estimates of energy intake: comparing three self-report measures to total energy expenditure from doubly labeled water. Annals of Epidemiology 2002; 12: 577-86.

29 Hebert JR, Ma Y, Clemow L, Ockene IS, Saperia G, Stanek EJ 3rd, et al. Gender differences in social desirability and social approval bias in dietary self-report. American Journal of Epidemiology 1997; 146: 1046-55.

30 Novotny JA, Rumpler WV, Riddick H, Hebert JR, Rhodes D, Judd JT, et al. Personality characteristics as predictors of underreporting of energy intake on 24-hour dietary recall interviews. Journal of the American Dietetic Association 2003; 103: 1146-51.

31 Hebert JR, Ebbeling CB, Ockene IS, Ma Y, Rider L, Merriam $\mathrm{PA}$, et al. A dietitian-delivered group nutrition program leads to reductions in dietary fat, serum cholesterol, and body weight: the Worcester Area Trial for Counseling in Hyperlipidemia (WATCH). Journal of the American Dietetic Association 1999; 99: 544-52.

32 Rosell MS, Hellenius ML, de Faire UH, Johansson GK. Associations between diet and the metabolic syndrome vary with the validity of dietary intake data. American Journal of Clinical Nutrition 2003; 78: 84-90.

33 Macdiarmid JI, Vail A, Cade JE, Blundell JE. The sugar-fat relationship revisited: differences in consumption between men and women of varying BMI. International Journal of Obesity and Related Metabolic Disorders 1998; 22: 1053-61. 